PROCEEDINGS OF THE

AMERICAN MATHEMATICAL SOCIETY

Volume 137, Number 11, November 2009, Pages 3609-3618

S 0002-9939(09)09942-0

Article electronically published on June 10, 2009

\title{
BOSONIC REALIZATION OF TOROIDAL LIE ALGEBRAS OF CLASSICAL TYPES
}

\author{
NAIHUAN JING, KAILASH C. MISRA, AND CHONGBIN XU \\ (Communicated by Gail R. Letzter)
}

\begin{abstract}
Generalizing Feingold and Frenkel's construction, we use Weyl bosonic fields to construct toroidal Lie algebras of types $A_{n}, B_{n}, C_{n}$ and $D_{n}$ of levels $-1,-2,-1 / 2$ and -2 respectively. In particular, our construction also gives new bosonic constructions for orthogonal Lie algebras in the cases of affine Lie algebras.
\end{abstract}

\section{INTRODUCTION}

Toroidal Lie algebras are natural generalizations of the affine Kac-Moody algebras MRY that enjoy many similar interesting features. Let $\mathfrak{g}$ be a finitedimensional complex simple Lie algebra of type $X_{n}$, and let $R=\mathbb{C}\left[s, s^{-1}, t, t^{-1}\right]$ be the ring of Laurent polynomials in commuting variables $s$ and $t$. By definition, a 2-toroidal Lie algebra of type $X_{n}$ is a perfect central extension of the iterated loop algebra $\mathfrak{g} \otimes R$ and can be realized as a certain homomorphic image of the universal central extension $T\left(X_{n}\right)=(g \otimes R) \oplus \Omega_{R} / d R$, where $\Omega_{R} / d R$ is the Kähler differential of $R$ modulo the exact forms. The center contains two special elements, $c_{0}$ and $c_{1}$. A module of $T\left(X_{n}\right)$ is called a level- $\left(k_{0}, k_{1}\right)$ module if the standard pair of central elements $\left(c_{0}, c_{1}\right)$ acts as $\left(k_{0}, k_{1}\right)$ for some complex numbers $k_{0}$ and $k_{1}$. In this work we will focus on modules with $k_{0} \neq 0$.

Two-toroidal Lie algebras resemble affine Lie algebras in many aspects. Their main source of representations consists of vertex operators (cf. MRY, B, BBS). In T] the toroidal Lie algebra of type $B_{n}$ was constructed using fermionic operators (see also $[\mathrm{JMg})$, and in $\mathrm{G}$ two constructions were given for the extended affine Lie algebras of type $A_{n}$. In [FJW] the first author and collaborators used McKay correspondence to realize level one representations of toroidal Lie algebras of simply laced types. In the recent paper [JM] a unified fermionic construction of toroidal Lie algebras of classical types was given by extending the Feingold-Frenkel realization coupled with new ghost fields. We pointed out there that the fermionic construction cannot be directly generalized to symplectic toroidal Lie algebras as one needs bosons to realize the long root vector $2 \varepsilon_{n}$.

Received by the editors November 19, 2008, and, in revised form, February 23, 2009.

2000 Mathematics Subject Classification. Primary 17B60, 17B67, 17B69; Secondary 17A45, $81 \mathrm{R} 10$.

Key words and phrases. Toroidal algebras, Weyl algebras, vertex operators, representations.

The first author was supported by NSA grant H98230-06-1-0083 and NSFC grant 10728102, and the second author was supported by NSA grant H98230-08-0080.

(C)2009 American Mathematical Society Reverts to public domain 28 years from publication 
In this paper we give a unified Weyl bosonic construction of all classical toroidal Lie algebras. Our results for types $C$ and $A$ are toroidal analogs of the FeingoldFrenkel construction $[\mathrm{FF}$. for affine Lie algebras (see also $[\mathrm{L}$ for a recent treatment of the Feingold-Frenkel construction). We also construct bosonic realizations for types $D$ and $B$. This includes, as special cases, new constructions (at level -2 ) for orthogonal affine Lie algebras. The main idea is again similar to that of [JM] to construct certain operators corresponding to the special nodes in the affine Dynkin diagrams. The novel results are specialized to the cases of toroidal Lie algebras. In a sense we have also obtained field operators corresponding to imaginary root vectors.

The Weyl bosonic construction for orthogonal toroidal Lie algebras is not directly obtained by mimicking the construction for type $A$ or type $C$, and we have to use a new embedding of the Lie algebras into type $A$ to achieve the goal, which partly explains why this construction has been missed in previous constructions of affine Lie algebras. Like the Neveu-Schwarz and Ramond fields in the fermionic case, our field operators have two forms indexed by half-integers and integers respectively.

The structure of the paper is as follows. In section 2 we define the toroidal Lie algebra and state the MRY presentation MRY of the toroidal algebra in terms of generators and relations. In section 3 we start with a finite rank lattice with an anti-symmetric bilinear form and define a Fock space and some bosonic field (vertex) operators, which in turn give level -1 representations of the toroidal Lie algebras of type $A_{n}$, level $-1 / 2$ modules for type $C$, and level -2 modules for types $B_{n}$ and $D_{n}$. The proof consists of an extensive analysis of the operator product expansions for the field operators. We also include the verification of the Serre relations.

\section{TOROIDAL Lie ALGEBraS}

A special quotient algebra of the toroidal Lie algebra $T\left(X_{n}\right)$ is the double affine algebra, denoted by $T_{0}\left(X_{n}\right)$, which is the toroidal Lie algebra of type $X_{n}$ with a two dimensional center. The double affine algebra is the quotient of $T\left(X_{n}\right)$ modulo all the central elements with degree other than zero. In fact, $T_{0}\left(X_{n}\right)$ has the following realization:

$$
T_{0}\left(X_{n}\right)=(\mathfrak{g} \otimes R) \oplus \mathbb{C} c_{0} \oplus \mathbb{C} c_{1},
$$

where $R$ is a ring and the Lie product is

$$
\left[x \otimes g_{1}, y \otimes g_{2}\right]=[x, y] \otimes g_{1} g_{2}+\Phi\left(g_{2} \partial_{s} g_{1}\right) c_{0}+\Phi\left(g_{2} \partial_{t} g_{1}\right) c_{1}
$$

for all $x, y \in \mathfrak{g}$ and $g_{1}, g_{2} \in R$, where $\Phi$ is a linear functional on $R$ defined by $\Phi\left(s^{k} t^{m}\right)=0$ if $(k, m) \neq(0,0)$ and $\Phi\left(s^{k} t^{m}\right)=1$ if $(k, m)=(0,0)$ for all $k, m \in \mathbb{Z}$.

For our purpose we will need the formal power series in variables $z$ and $w$. In particular we will need the formal delta functions $\delta(z-w)=\sum_{n \in \mathbb{Z}} z^{-n-1} w^{n}$. This can be understood as follows:

$$
\begin{aligned}
\delta(z-w) & =\iota_{z, w}\left((z-w)^{-1}\right)+\iota_{w, z}\left((w-z)^{-1}\right), \\
\partial_{w} \delta(z-w) & =\iota_{z, w}\left((z-w)^{-2}\right)-\iota_{w, z}\left((w-z)^{-2}\right),
\end{aligned}
$$

where $\iota_{z, w}$ means expansion when $|z|>|w|$. For simplicity, in the following we will drop $\iota_{z, w}$ if it is clear from the context.

For $n \geq 1$ let $\left(a_{i j}\right)_{n+1 \times n+1}$ be the generalized Cartan matrix of the affine algebra $X_{n}^{(1)}$ and $Q:=\mathbb{Z} \alpha_{0} \oplus \mathbb{Z} \alpha_{1} \oplus \cdots \oplus \mathbb{Z} \alpha_{n}$ its root lattice. The toroidal Lie algebra $T\left(X_{n}\right)$ 
[MRY] is the Lie algebra generated by $\not k, \alpha_{i}(k)$ and $x_{k}\left( \pm \alpha_{i}\right)$ for $i=0,1, \cdots, n$ and $k \in \mathbb{Z}$, with the following relations:

$(R 0)\left[k, \alpha_{i}(z)\right]=0=\left[k, x\left( \pm \alpha_{i}, z\right)\right]$

$(R 1)\left[\alpha_{i}(z), \alpha_{j}(w)\right]=\left(\alpha_{i} \mid \alpha_{j}\right) \partial_{w} \delta(z-w) k$;

$(R 2)\left[\alpha_{i}(z), x\left( \pm \alpha_{j}, w\right)\right]= \pm\left(\alpha_{i} \mid \alpha_{j}\right) x\left( \pm \alpha_{j}, w\right) \delta(z-w)$;

$(R 3)\left[x\left(\alpha_{i}, z\right), x\left(-\alpha_{j}, w\right)\right]=\delta_{i j} \frac{2}{\left(\alpha_{i} \mid \alpha_{j}\right)}\left\{\alpha_{i}(w) \delta(z-w)+\partial_{w} \delta(z-w) \not k\right\}$;

(R4) $\left[x\left(\alpha_{i}, z\right), x\left(\alpha_{i}, w\right)\right]=0=\left[x\left(-\alpha_{i}, z\right), x\left(-\alpha_{i}, w\right)\right]$;

(S1) $\operatorname{ad} x\left( \pm \alpha_{i}, z_{1}\right) x\left( \pm \alpha_{j}, z_{2}\right)=0, \quad$ for $a_{i j}=0$;

$(S 2)\left(\operatorname{ad} x\left( \pm \alpha_{i}, z_{1}\right)\right)\left(\operatorname{ad} x\left( \pm \alpha_{i}, z_{2}\right)\right) x\left( \pm \alpha_{j}, z_{3}\right)=0$, if $a_{i j}=-1$;

(S3) $\left(\operatorname{ad} x\left( \pm \alpha_{i}, z_{1}\right)\right)\left(\operatorname{ad} x\left( \pm \alpha_{i}, z_{2}\right)\right)\left(\operatorname{ad} x\left( \pm \alpha_{i}, z_{3}\right)\right) x\left( \pm \alpha_{j}, z_{4}\right)=0$, if $a_{i j}=-2$; where we have used the following power series:

$$
\begin{gathered}
\alpha_{i}(z)=\sum_{n \in \mathbb{Z}} \alpha_{i}(n) z^{-n-1}, \\
x\left( \pm \alpha_{i}, z\right)=\sum_{n \in \mathbb{Z}} x_{n}\left( \pm \alpha_{i}\right) z^{-n-1} .
\end{gathered}
$$

We remark that the 2-toroidal Lie algebra of type $X_{n}$ includes two affine Lie algebras of type $X_{n}^{(1)}$ as subalgebras.

\section{Representations of the toroidal Algebra}

In this section we give two unified bosonic realizations for the toroidal Lie algebras of classical types $A_{n-1}, B_{n}, C_{n}$ and $D_{n}$, using bosonic analogs of NeveuSchwarz and Ramond fermionic fields.

Let $\left\{\varepsilon_{i}\right\}(i=1, \ldots, n+1)$ be an orthonormal basis of the vector space $\mathbb{C}^{n+1}$ equipped with the inner product $(\mid)$ such that

$$
\left(\varepsilon_{i} \mid \varepsilon_{j}\right)=\delta_{i j}
$$

Let $P_{0}=\mathbb{Z} \varepsilon_{1} \oplus \cdots \oplus \mathbb{Z} \varepsilon_{n}$ be a sublattice of rank $n$, and let $\varepsilon_{0}^{\prime}$ be an external unit vector orthogonal to $\varepsilon_{i}$. Introduce $\bar{c}=\frac{1}{\sqrt{2}}\left(\varepsilon_{0}^{\prime}+i \varepsilon_{n+1}\right)$, corresponding to the null vector $\delta$, and let $\bar{d}=\frac{1}{\sqrt{2}}\left(\varepsilon_{0}^{\prime}-i \varepsilon_{n+1}\right)$ be the dual gradation operator. Then

$$
\begin{aligned}
(\bar{c} \mid \bar{c}) & =\left(\bar{c} \mid \varepsilon_{i}\right)=0, \\
(\bar{d} \mid \bar{d}) & =\left(\bar{d} \mid \varepsilon_{i}\right)=0, \quad(\bar{c} \mid \bar{d})=1
\end{aligned}
$$

for $i=1, \ldots, n$.

The simple roots for the classical finite dimensional Lie algebras can be realized simply by defining them as follows:

$$
\begin{aligned}
& \alpha_{1}=\varepsilon_{1}-\varepsilon_{2}, \cdots, \alpha_{n-1}=\varepsilon_{n-1}-\varepsilon_{n} \text { for } A_{n-1} ; \\
& \alpha_{1}=\varepsilon_{1}-\varepsilon_{2}, \cdots, \alpha_{n-1}=\varepsilon_{n-1}-\varepsilon_{n}, \alpha_{n}=\varepsilon_{n} \text { for } B_{n} ; \\
& \alpha_{1}=\frac{1}{\sqrt{2}}\left(\varepsilon_{1}-\varepsilon_{2}\right), \cdots, \alpha_{n-1}=\frac{1}{\sqrt{2}}\left(\varepsilon_{n-1}-\varepsilon_{n}\right), \alpha_{n}=\sqrt{2} \varepsilon_{n} \text { for } C_{n} ; \\
& \alpha_{1}=\varepsilon_{1}-\varepsilon_{2}, \cdots, \alpha_{n-1}=\varepsilon_{n-1}-\varepsilon_{n}, \alpha_{n}=\varepsilon_{n-1}+\varepsilon_{n} \text { for } D_{n} .
\end{aligned}
$$

Then the set of positive roots is:

$$
\Delta_{+}= \begin{cases}\left\{\varepsilon_{i}-\varepsilon_{j} \mid 1 \leq i<j \leq n\right\}, & \text { Type } A_{n-1} \\ \left\{\varepsilon_{i}, \varepsilon_{i} \pm \varepsilon_{j} \mid 1 \leq i<j \leq n\right\}, & \text { Type } B_{n} \\ \left\{\sqrt{2} \varepsilon_{i}, \frac{1}{\sqrt{2}}\left(\varepsilon_{i} \pm \varepsilon_{j}\right) \mid 1 \leq i<j \leq n\right\}, & \text { Type } C_{n} \\ \left\{\varepsilon_{i} \pm \varepsilon_{j} \mid 1 \leq i<j \leq n\right\}, & \text { Type } D_{n} .\end{cases}
$$


The highest (long) root $\alpha_{\max }$ for each type is given as follows:

$$
\alpha_{\max }= \begin{cases}\varepsilon_{1}-\varepsilon_{n}, & \text { Type } A_{n-1} \\ \varepsilon_{1}+\varepsilon_{2}, & \text { Type } B_{n} \text { or } D_{n} \\ \sqrt{2} \varepsilon_{1}, & \text { Type } C_{n} .\end{cases}
$$

We further introduce the element

$$
\alpha_{0}=\bar{c}-\alpha_{\max }
$$

in the lattice and then define $\beta=-\bar{c}+\varepsilon_{1}$ for type $A, B$, or $D$ and $\beta=-\sqrt{2} \bar{c}+\varepsilon_{1}$ for type $C$. Then we have

$$
\alpha_{0}=\varepsilon_{n}-\beta \text { for } A_{n-1}, \quad-\beta-\varepsilon_{2} \text { for } B_{n} \text { and } D_{n}, \quad \text { or }-\frac{1}{\sqrt{2}}\left(\beta+\varepsilon_{1}\right) \text { for } C_{n} .
$$

Note that $(\beta \mid \beta)=1,\left(\beta \mid \varepsilon_{i}\right)=\delta_{1 i}$.

Then $P=\mathbb{Z} \bar{c} \oplus \mathbb{Z} \varepsilon_{1} \oplus \cdots \oplus \mathbb{Z} \varepsilon_{n}$ and $Q=\mathbb{Z} \bar{c} \oplus \mathbb{Z} \alpha_{1} \oplus \cdots \oplus \mathbb{Z} \alpha_{k}=\mathbb{Z} \alpha_{0} \oplus \cdots \oplus \mathbb{Z} \alpha_{k}$ $\left(k=n-1\right.$ for type $A_{n-1}$ and $k=n$ for types $B_{n}, C_{n}$ and $D_{n}$ ) are the weight lattice and root lattice for the corresponding affine Lie algebra, and $P_{0}=\mathbb{Z} \varepsilon_{1} \oplus \cdots \oplus \mathbb{Z} \varepsilon_{n}$ and $Q_{0}=\mathbb{Z} \alpha_{1} \oplus \cdots \oplus \mathbb{Z} \alpha_{k}\left(k=n-1\right.$ for type $A_{n-1}$ and $k=n$ for types $B_{n}, C_{n}$ and $\left.D_{n}\right)$ are the weight lattice and root lattice for the simple Lie algebras $A_{n-1}, B_{n}, C_{n}$ and $D_{n}$. Then $\left(\alpha_{i} \mid \alpha_{j}\right)=d_{i} a_{i j}$, where $a_{i j}$ are the entries of the affine Cartan matrix of type $(A B C D)^{(1)}$ and the $d_{i}$ 's are given by

$$
\left(d_{0}, d_{1}, \cdots, d_{k}\right)= \begin{cases}\{1,1, \cdots, 1,1\}, & k=n-1, \text { Type } A_{n-1} \\ \left\{1,1, \cdots, 1, \frac{1}{2}\right\}, & k=n, \text { Type } B_{n} \\ \left\{1, \frac{1}{2}, \cdots, \frac{1}{2}, 1\right\}, & k=n, \text { Type } C_{n} \\ \{1,1, \cdots, 1,1\}, & k=n, \text { Type } D_{n} .\end{cases}
$$

Note that the bilinear form $(\mid)$ is non-degenerate on the affine root lattice or the span of $\{\beta\} \cup\left\{\alpha_{i}\right\}(i \geq 1)$.

We introduce infinite dimensional Weyl algebras as follows. Let $\mathbf{Z}=\mathbb{Z}$ or $\mathbf{Z}=$ $\mathbb{Z}+1 / 2$. Let $P_{\mathbb{C}}=P \otimes \mathbb{C}$ be the $\mathbb{C}$-vector space spanned by $\bar{c}$ and $\varepsilon_{i}, 1 \leq i \leq n$, in the various types. To combine all types we will also introduce another set of orthonormal vectors $\varepsilon_{\bar{i}}, 1 \leq i \leq n+1$, such that

$$
\left(\varepsilon_{\bar{i}} \mid \varepsilon_{\bar{j}}\right)=\delta_{i j}
$$

and we denote by $\bar{P}_{\mathbb{C}}$ the $\mathbb{C}$-space spanned by $\varepsilon_{\bar{i}}$. We also define $\bar{\beta}=-\bar{c}+\varepsilon_{\overline{1}}$ and $\alpha_{\overline{0}}=\bar{c}-\alpha_{\overline{m a x}}$, where $\alpha_{\overline{m a x}}=\varepsilon_{\overline{1}}+\varepsilon_{\overline{2}}$. The special vectors are only needed in types $B_{n}$ and $D_{n}$, and $\varepsilon \overline{n+1}$ is only needed for type $B_{n}$. We define $\mathcal{C}=\mathcal{C}_{1} \oplus \mathcal{C}_{2}$, with both subspaces $\mathcal{C}_{1}=P_{\mathbb{C}} \oplus \bar{P}_{\mathbb{C}}$ and $\mathcal{C}_{2}=P_{\mathbb{C}}^{*} \oplus \bar{P}_{\mathbb{C}}^{*}$ being maximal isotropic subspaces, where $\langle P, \bar{P}\rangle=0$. We define the natural anti-symmetric bilinear form on $\mathcal{C}$ by

$$
\left\langle b^{*}, a\right\rangle=-\left\langle a, b^{*}\right\rangle=(a \mid b), \quad\langle a, b\rangle=\left\langle a^{*}, b^{*}\right\rangle=0, \quad a, b \in \mathcal{C}_{1} .
$$

The Weyl algebra $W(P)$ is the unital algebra generated by the elements $a(k)$ and $a^{*}(k)$, where $a \in \mathcal{C}_{1}, a^{*} \in \mathcal{C}_{2}$ and $k \in \mathbf{Z}$, subject to the relations

$$
[u(k), v(l)]=\langle u, v\rangle \delta_{k,-l}, \quad u, v \in \mathcal{C} .
$$

The representation space is the infinite dimensional vector space

$$
V=\bigotimes_{a_{i}}\left(\bigotimes_{k \in \mathbf{Z}_{+}} \mathbb{C}\left[a_{i}(-k)\right] \bigotimes_{k \in \mathbf{Z}_{+}} \mathbb{C}\left[a_{i}^{*}(-k)\right]\right)
$$


where $a_{i}$ runs through any basis in $P$ and $\bar{P}$, consisting of, say, $\bar{c}, \varepsilon_{i}$ 's and $\varepsilon_{\bar{i}}$ 's.

The Weyl algebra acts on the space $V$ by the usual action: $a(-k)$ acts as a creation operator and $a(k)$ as an annihilation operator. We remark that using $-\langle$,$\rangle as the form on \mathcal{C}$ is equivalent to switching $\mathcal{C}_{1}$ and $\mathcal{C}_{2}$. As the symplectic form $\langle$,$\rangle is non-degenerate, the Fock space V$ is an irreducible module for the Weyl algebra.

For any bosonic fields

$$
u(z)=\sum_{n \in \mathbf{Z}} u(n) z^{-n-1 / 2}, \quad v(z)=\sum_{n \in \mathbf{Z}} v(n) z^{-n-1 / 2}
$$

we define the normal ordering : $u(z) v(w)$ : by swapping their components, i.e.

$$
: u(m) v(n):= \begin{cases}u(m) v(n), & m<0 \\ \frac{1}{2}(u(0) v(n)+v(n) u(0)), & m=0 \\ v(n) u(m), & m>0 .\end{cases}
$$

It then follows that $: u(z) v(w):=: v(w) u(z):$.

Based on the normal product of two fields, we can define the normal product of $n$ fields inductively as follows. In fact we define

$$
: u_{1}\left(z_{1}\right) u_{2}\left(z_{2}\right) \cdots u_{n}\left(z_{n}\right):=: u_{1}\left(z_{1}\right)\left(: u_{2}\left(z_{2}\right) \cdots u_{n}\left(z_{n}\right):\right):
$$

and then use induction till we reach two fields.

We define the contraction of two states by

$$
\underbrace{a(z) b(w)}=a(z) b(w)-: a(z) b(w):
$$

which contains all poles for $a(z) b(w)$. In general, the contraction of several pairs of states is given inductively by the following rule.

Theorem 3.1 (Wick's theorem $[\mathrm{FF}, \mathrm{K}]$ ). For elements $v_{1}, \ldots, v_{n}$ we have

$$
\begin{aligned}
& : u_{1} \cdots u_{m}:: v_{1} \cdots v_{n}:=: u_{1} \cdots u_{m} v_{1} \cdots v_{n}:+ \\
& \sum_{i_{1}<\cdots<i_{s}} \underbrace{u_{i_{1}} v_{j_{1}}} \cdots \underbrace{u_{i_{s}} v_{j_{s}}}: u_{1} \cdots \hat{u}_{i_{1}} \cdots \hat{u}_{i_{s}} \cdots u_{m} v_{1} \cdots \hat{v}_{j_{1}} \cdots \hat{v}_{j_{s}} \cdots v_{n}:
\end{aligned}
$$

where the sum runs over all possible contractions of some $u_{i}$ 's and some $v_{i}$ 's.

Proposition 3.1. The basic operator product expansions are: for $u, v \in \mathcal{C}$,

$$
\underbrace{u(z) v(w)}=\frac{\langle u, v\rangle}{z-w} \text { for } \mathbb{Z}+1 / 2, \quad\langle u, v\rangle \frac{(z+w)(z w)^{-1 / 2}}{2(z-w)} \text { for } \mathbb{Z} .
$$

In particular we have, for $a, b \in P_{\mathbb{C}}$ and $\mathbb{Z}+1 / 2$,

$$
\begin{aligned}
& \underbrace{a(z) b(w)}=\underbrace{a^{*}(z) b^{*}(w)}_{\left\langle a, b^{*}\right\rangle}=0, \\
& \underbrace{a(z) b^{*}(w)}=\frac{\left\langle a^{*}(z) b(w)\right.}{z-w}=\frac{\left\langle a^{*}, b\right\rangle}{z-w} .
\end{aligned}
$$


Proof. In fact one has

$$
\begin{aligned}
\underbrace{a(z) b^{*}(w)} & =\frac{\left\langle a, b^{*}\right\rangle}{2} z^{-1 / 2} w^{-1 / 2} \delta_{\mathbf{Z}, \mathbb{Z}}+\sum_{n \in \mathbf{Z}, 0<m \in \mathbf{Z}}\left[a(m), b^{*}(n)\right] z^{-m-1 / 2} w^{-n-1 / 2} \\
& =\left\langle a, b^{*}\right\rangle\left(\frac{1}{2} z^{-1 / 2} w^{-1 / 2} \delta_{\mathbf{Z}, \mathbb{Z}}+\sum_{0<m \in \mathbf{Z}} z^{-m-1 / 2} w^{m-1 / 2}\right) \\
& = \begin{cases}\frac{\left\langle a, b^{*}\right\rangle}{z-w}, & \mathbf{Z}=\mathbb{Z}+1 / 2 \\
\left\langle a, b^{*}\right\rangle \frac{(z+w)(z w)^{-1 / 2}}{2(z-w)}, & \mathbf{Z}=\mathbb{Z} .\end{cases}
\end{aligned}
$$

The other operator product expansions are proved in the same manner.

Note that in both cases $(\mathbb{Z}$ and $\mathbb{Z}+1 / 2)$ we have the following result.

Proposition 3.2. The bosonic fields satisfy the following commutation relation:

$$
[u(z), v(w)]=\langle u, v\rangle \delta(z-w) .
$$

Proof. Using the fact that : $u(z) v(w):=: v(w) u(z):$, it follows from Proposition 3.1 that

$$
\begin{aligned}
{[u(z), v(w)] } & =u(z) v(w)-v(w) u(z) \\
& =\langle u, v\rangle \delta(z-w) .
\end{aligned}
$$

Proposition 3.3. The commutators among normal order products are given by

$$
\begin{aligned}
& {\left[: a_{1}(z) a_{2}^{*}(z):,: b_{1}(w) b_{2}^{*}(w):\right]=\left\langle a_{1}, b_{2}^{*}\right\rangle: a_{2}^{*}(z) b_{1}(z): \delta(z-w)} \\
& \quad+\left\langle a_{2}^{*}, b_{1}\right\rangle: a_{1}(z) b_{2}^{*}(z): \delta(z-w)+\left\langle a_{1}, b_{2}^{*}\right\rangle\left\langle a_{2}^{*}, b_{1}\right\rangle \partial_{w} \delta(z-w) .
\end{aligned}
$$

Proof. By Theorem 3.1 and Proposition 3.1, for $\mathbb{Z}+1 / 2$ we have

$$
\begin{aligned}
& : a_{1}(z) a_{2}^{*}(z):: b_{1}(w) b_{2}^{*}(w):=: a_{1}(z) a_{2}^{*}(z) b_{1}(z) b_{2}^{*}(w): \\
& \quad+\frac{\left\langle a_{1}, b_{2}^{*}\right\rangle}{z-w}: a_{2}^{*}(z) b_{1}(w):+\frac{\left\langle a_{2}^{*}, b_{1}\right\rangle}{z-w}: a_{1}(z) b_{2}^{*}(w):+\frac{\left\langle a_{1}, b_{2}^{*}\right\rangle\left\langle a_{2}^{*}, b_{1}\right\rangle}{(z-w)^{2}} .
\end{aligned}
$$

Hence the result follows. The case of $\mathbb{Z}$ is shown similarly.

The (anti-symmetric) inner product of the underlying Lie algebra can be extended to an inner product of the linear factors as follows:

$$
\left\langle: r_{1} r_{2}:,: s_{1} s_{2}:\right\rangle=-\left\langle r_{1}, s_{1}\right\rangle\left\langle r_{2}, s_{2}\right\rangle+\left\langle r_{1}, s_{2}\right\rangle\left\langle r_{2}, s_{1}\right\rangle \text {. }
$$

In the following we will describe the bosonic realization of toroidal Lie algebras of classical types.

(i) Type $A_{n-1}(n \geq 2)$. The roots of the finite dimensional Lie algebra are $\varepsilon_{i}-\varepsilon_{j}$ $(1 \leq i \neq j \leq n)$. We associate to each finite root and each imaginary root $\delta$ the following field operators. The level is $\not k=-1$.

$$
\begin{aligned}
X\left(\varepsilon_{i}-\varepsilon_{j}, z\right) & =: \varepsilon_{i}(z) \varepsilon_{j}^{*}(z):, \quad h_{\varepsilon_{i}-\varepsilon_{j}}(z)=: \varepsilon_{i}(z) \varepsilon_{i}^{*}(z):-: \varepsilon_{j}(z) \epsilon_{j}^{*}(z): \\
X\left( \pm \alpha_{i}, z\right) & =X\left( \pm\left(\varepsilon_{i}-\varepsilon_{i+1}\right), z\right), \quad \alpha_{i}(z)=h_{\varepsilon_{i}-\varepsilon_{i+1}}(z) \\
X\left(\alpha_{0}, z\right) & =: \varepsilon_{n}(z) \beta^{*}(z):, \quad X\left(-\alpha_{0}, z\right)=: \beta(z) \varepsilon_{n}^{*}(z): \\
a_{0}(z) & =: \varepsilon_{n}(z) \varepsilon_{n}^{*}(z):-: \beta(z) \beta^{*}(z):
\end{aligned}
$$


Using Proposition 3.3 we get, for $i \neq j$,

$$
\begin{gathered}
{\left[X\left(\varepsilon_{i}-\varepsilon_{j}, z\right), X\left(\varepsilon_{k}-\varepsilon_{l}, w\right)\right]=\delta_{j k} X\left(\varepsilon_{i}-\varepsilon_{l}, z\right) \delta(z-w)} \\
-\delta_{l i} X\left(\varepsilon_{k}-\varepsilon_{j}, z\right) \delta(z-w)-\delta_{j k} \delta_{l i} \partial_{w} \delta(z-w), \\
{\left[X\left(\varepsilon_{i}-\varepsilon_{j}, z\right), X\left(\varepsilon_{j}-\varepsilon_{i}, w\right)\right]=\delta_{i j}\left(h_{\varepsilon_{i}-\varepsilon_{j}}(z) \delta(z-w)-\partial_{w} \delta(z-w)\right),} \\
{\left[h_{\varepsilon_{i}-\varepsilon_{j}}(z), X\left(\varepsilon_{k}-\varepsilon_{l}, w\right)\right]=\left(\varepsilon_{i}-\varepsilon_{j} \mid \varepsilon_{k}-\varepsilon_{l}\right) X\left(\varepsilon_{j}-\varepsilon_{k}, w\right) \delta(z-w),}
\end{gathered}
$$

where $\varepsilon_{i}, \varepsilon_{j}$ can be $\beta$ (recall that $\left.\left(\beta \mid \varepsilon_{i}\right)=\delta_{1 i}\right)$. These commutation relations show that the construction provides a level -1 realization of the affine Lie algebra of type $A$. Moreover they provide a representation for the toroidal Lie algebra, as the commutation relations involving $\alpha_{0}$ are similar to the above. In fact,

$$
\begin{aligned}
& {\left[X\left(\alpha_{0}, z\right), X\left(\alpha_{1}, w\right)\right]=: \varepsilon_{n}(z) \varepsilon_{2}^{*}(z): \delta(z-w), \quad\left[X\left(\alpha_{0}, z\right), X\left(-\alpha_{1}, w\right)\right]=0,} \\
& {\left[X\left(\alpha_{0}, z\right), X\left(\alpha_{n}, w\right)\right]=-: \beta^{*}(z) \varepsilon_{n-1}(z): \delta(z-w), \quad\left[X\left(\alpha_{0}, z\right), X\left(-\alpha_{n}, w\right)\right]=0 .}
\end{aligned}
$$

(ii) Type $C_{n}(n \geq 2)$. The finite roots are $\alpha_{i j}=\frac{1}{\sqrt{2}}\left(\varepsilon_{i}-\varepsilon_{j}\right)(1 \leq i \neq j \leq n)$, $\pm \beta_{i j}= \pm \frac{1}{\sqrt{2}}\left(\varepsilon_{i}+\varepsilon_{j}\right)(1 \leq i \neq j \leq n)$. Note that $-\alpha_{i j}=\alpha_{j i}$. We realize the toroidal Lie algebra by the following field operators, and the level is $k=-1 / 2$.

$$
\begin{aligned}
X\left(\alpha_{i j}, z\right) & =: \varepsilon_{i}(z) \varepsilon_{j}^{*}(z):, \quad h_{\varepsilon_{i}-\varepsilon_{j}}(z)=: \varepsilon_{i}(z) \varepsilon_{i}^{*}(z):-: \varepsilon_{j}(z) \varepsilon_{j}^{*}(z):, \\
X\left(\beta_{i j}, z\right) & =: \varepsilon_{i}(z) \varepsilon_{j}(z):, \quad X\left(-\beta_{i j}, z\right)=-: \varepsilon_{i}^{*}(z) \varepsilon_{j}^{*}(z):, \\
h_{\varepsilon_{i}+\varepsilon_{j}}(z) & =: \varepsilon_{i}(z) \varepsilon_{i}^{*}(z):+: \varepsilon_{j}(z) \varepsilon_{j}^{*}(z):, \\
X\left( \pm \alpha_{i}, z\right) & =X\left( \pm \alpha_{i, i+1}, z\right), \quad \alpha_{i}(z)=(1 / 2) h_{\varepsilon_{i}-\varepsilon_{i+1}}(z), \\
X\left( \pm \alpha_{n}, z\right) & =(1 / 2) X\left( \pm \beta_{n n}, z\right), \quad \alpha_{n}(z)=h_{2 \varepsilon_{n}}(z), \\
X\left(\alpha_{0}, z\right) & =(1 / 2): \beta^{*}(z) \varepsilon_{1}^{*}(z):, \quad X\left(-\alpha_{0}, z\right)=(1 / 2): \beta(z) \varepsilon_{1}(z):, \\
\alpha_{0}(z) & =(1 / 4)\left(: \varepsilon_{1}(z) \varepsilon_{1}^{*}(z):+: \beta(z) \beta^{*}(z):+: \beta^{*}(z) \varepsilon_{1}(z):+: \varepsilon_{1}^{*}(z) \beta(z):\right) .
\end{aligned}
$$

Several commutation relations are exactly the same as for type $A$. Some new ones are obtained by Proposition 3.3 as follows:

$$
\begin{aligned}
& {\left[X\left(\beta_{i j}, z\right), X\left(-\beta_{k l}, w\right)\right]} \\
& \quad=\left(\delta_{i k} X\left(\alpha_{j l}, z\right)+\delta_{i l} X\left(\alpha_{j k}, z\right)+\delta_{j k} X\left(\alpha_{j l}, z\right)\right. \\
& \left.\quad+\delta_{j l} X\left(\alpha_{i k}, z\right)\right) \delta(z-w)-\left(\delta_{i k} \delta_{j l}+\delta_{i k} \delta_{j l}\right) \partial_{w} \delta(z-w) \\
& {\left[X\left(\alpha_{i j}, z\right), X\left(\beta_{k l}, w\right)\right]=\left(\delta_{j k} X\left(\beta_{i l}, z\right)+\delta_{j l} X\left(\beta_{i k}, z\right)\right) \delta(z-w),} \\
& {\left[X\left(\alpha_{i j}, z\right), X\left(-\beta_{k l}, w\right)\right]=-\left(\delta_{i k} X\left(-\beta_{j l}, z\right)+\delta_{i l} X\left(-\beta_{j k}, z\right)\right) \delta(z-w) .}
\end{aligned}
$$

(iii) Type $D_{n}(n \geq 4)$. The finite roots are $\pm \varepsilon_{i} \pm \varepsilon_{j}(1 \leq i \neq j \leq n)$. We realize the toroidal Lie algebra by the following field operators. The level is $k=-2$. For 
$i<j$ we define

$$
\begin{aligned}
X\left(\varepsilon_{i}-\varepsilon_{j}, z\right) & =: \varepsilon_{i}(z) \varepsilon_{j}^{*}(z):-: \varepsilon_{\bar{j}}(z) \varepsilon_{i}^{*}(z): \\
h_{\varepsilon_{i}-\varepsilon_{j}}(z) & =: \varepsilon_{i}(z) \varepsilon_{i}^{*}(z):-: \varepsilon_{j}(z) \varepsilon_{j}^{*}(z):-: \varepsilon_{\bar{i}}(z) \varepsilon_{\bar{i}}^{*}(z):+: \varepsilon_{\bar{j}}(z) \varepsilon_{\bar{j}}^{*}(z): \\
X\left(\varepsilon_{i}+\varepsilon_{j}, z\right) & =: \varepsilon_{i}(z) \varepsilon_{\bar{j}}^{*}(z):-: \varepsilon_{j}(z) \varepsilon_{\bar{i}}^{*}(z):, \\
X\left(-\varepsilon_{i}-\varepsilon_{j}, z\right) & =: \varepsilon_{\bar{j}}(z) \varepsilon_{i}^{*}(z):-: \varepsilon_{\bar{i}}(z) \varepsilon_{j}^{*}(z):, \\
h_{\varepsilon_{i}+\varepsilon_{j}}(z) & =: \varepsilon_{i}(z) \varepsilon_{i}^{*}(z):+: \varepsilon_{j}(z) \varepsilon_{j}^{*}(z):-: \varepsilon_{\bar{i}}(z) \varepsilon_{\bar{i}}^{*}(z):-: \varepsilon_{\bar{j}}(z) \varepsilon_{\bar{j}}^{*}(z): \\
X\left( \pm \alpha_{i}, z\right) & =X\left( \pm\left(\varepsilon_{i}-\varepsilon_{i+1}\right), z\right), \quad \alpha_{i}(z)=h_{\varepsilon_{i}-\varepsilon_{i+1}}(z), \\
X\left( \pm \alpha_{n}, z\right) & =X\left( \pm\left(\varepsilon_{n-1}+\varepsilon_{n}\right), z\right), \quad \alpha_{n}(z)=h_{\varepsilon_{n-1}-\varepsilon_{n}}(z), \\
X\left(\alpha_{0}, z\right) & =: \varepsilon_{\overline{2}}(z) \beta^{*}(z):-: \bar{\beta}(z) \varepsilon_{2}^{*}(z):, \\
X\left(-\alpha_{0}, z\right) & =: \beta(z) \varepsilon_{\frac{2}{2}}(z):-: \varepsilon_{2}(z) \bar{\beta}^{*}(z):, \\
\alpha_{0}(z) & =: \varepsilon_{\overline{2}}(z) \varepsilon_{\overline{2}}^{*}(z):-: \varepsilon_{2}(z) \varepsilon_{2}^{*}(z):+: \bar{\beta}(z) \bar{\beta}^{*}(z):-: \beta^{*}(z) \beta(z): .
\end{aligned}
$$

(iv) Type $B_{n}(n \geq 2)$. The finite roots are $\pm \varepsilon_{i} \pm \varepsilon_{j}(1 \leq i \neq j \leq n)$ and $\pm \varepsilon_{i}$ $(1 \leq i \leq n)$. The level is $k=-2$. We realize the toroidal Lie algebra by the following field operators. The field operators for the roots $\pm \varepsilon_{i} \pm \varepsilon_{j}(i \neq j)$ are the same as for type $D_{n}$, though we need to add the bosonic fields $\varepsilon_{n+1}(z)$ and $\varepsilon_{n+1}^{*}(z)$ in the realization,

$$
\begin{aligned}
X\left(\varepsilon_{i}, z\right) & =: \varepsilon_{i}(z) \varepsilon_{\frac{*}{n+1}}^{*}(z):-: \varepsilon_{\overline{n+1}}(z) \varepsilon_{\bar{i}}^{*}(z):, \\
X\left(-\varepsilon_{i}, z\right) & =: \varepsilon_{\overline{n+1}}(z) \varepsilon_{i}^{*}(z):-: \varepsilon_{\bar{i}}(z) \varepsilon_{\overline{n+1}}^{*}(z):, \\
h_{\varepsilon_{i}}(z) & =: \varepsilon_{i}(z) \varepsilon_{i}^{*}(z):-: \varepsilon_{\bar{i}}(z) \varepsilon_{\bar{i}}^{*}(z): \\
X\left( \pm \alpha_{n}, z\right) & =X\left( \pm \varepsilon_{n}, z\right), \quad \alpha_{n}(z)=h_{\varepsilon_{n}}(z),
\end{aligned}
$$

and $X\left( \pm \alpha_{i}, x\right), \alpha_{i}(z)(0 \leq i \leq n-1)$ are given by the same formulae as in (iii). Using the same analysis we find that

$$
\begin{aligned}
{\left[X\left(\varepsilon_{i}, z\right), X\left(-\varepsilon_{j}, w\right)\right] } & =X\left(\varepsilon_{i}-\varepsilon_{j}, z\right) \delta(z-w)-2 \delta_{i j} \partial_{w} \delta(z-w), \\
{\left[X\left(\varepsilon_{i}, z\right), X\left(\varepsilon_{j}, w\right)\right] } & =-X\left(\varepsilon_{i}+\varepsilon_{j}, z\right) \delta(z-w) .
\end{aligned}
$$

Theorem 3.2. The previous constructions give rise to realizations of classical toroidal Lie algebras of types $A_{n-1}(n \geq 2), C_{n}(n \geq 2), D_{n}(n \geq 4)$ and $B_{n}(n \geq 3)$ with levels $-1,-1 / 2,-2$ and -2 respectively.

Proof. In the above construction we have outlined the major commutation relations of various types. One can use $d_{i}$ to combine the arguments for types $A_{n-1}$ and $C_{n}$. We will write $\alpha_{i j}=d_{i}^{-1 / 2}\left(\varepsilon_{i}-\varepsilon_{j}\right)=-\alpha_{j i}$ and $\beta_{i j}=d_{i}^{-1 / 2}\left(\varepsilon_{i}+\varepsilon_{j}\right)$ for $i \neq j$; then both types have the finite positive simple $\alpha_{i, i+1}$ plus $\beta_{n, n}$ in type $C_{n}$. First we note that for $i \neq j, k \neq l$,

$$
\begin{aligned}
{\left[: \varepsilon_{i}(z) \varepsilon_{j}^{*}(z):,: \varepsilon_{k}(w) \varepsilon_{l}^{*}(w):\right]=\left(\delta_{j k}: \varepsilon_{i}(z) \varepsilon_{l}^{*}(z):\right.} & \\
& \left.-\delta_{i l}: \varepsilon_{j}^{*}(z) \varepsilon_{k}(z):\right) \delta(z-w)-\delta_{i l} \delta_{j k} \partial_{w} \delta(z-w) .
\end{aligned}
$$

When $i=l$ and $j=k$ the identity reduces to

$$
\left[X\left(\alpha_{i j}, z\right), X\left(-\alpha_{i j}, w\right)\right]=d_{i}^{-1}\left(a_{i j}(z) \delta(z-w)-d_{i} \partial_{w} \delta(z-w)\right),
$$

which shows that the center $\not c$ is $-d_{i}$ for types $A_{n-1}$ and $C_{n}$. Here we also put

$$
a_{i j}(z)=d_{i}\left(: \varepsilon_{i}(z) \varepsilon_{i}^{*}(z):-: \varepsilon_{j}(z) \varepsilon_{j}^{*}(z):\right) .
$$


Then the Heisenberg field operators are generated by the simple ones, $\alpha_{i}(z)=$ $a_{i, i+1}(z)$. We then have by using (3.7) that

$$
\begin{aligned}
{\left[\alpha_{i}(z), \alpha_{j}(w)\right] } & =-d_{i}^{2}\left(2 \delta_{i j}-\delta_{i+1, j}-\delta_{i, j+1}\right) \partial_{w} \delta(z-w) \\
& =-d_{i}\left(\alpha_{i} \mid \alpha_{j}\right) \partial_{w} \delta(z-w) .
\end{aligned}
$$

Also, for any $k \neq l$ we find using (3.7) that

$$
\begin{aligned}
& {\left[\alpha_{i}(z), X\left(\alpha_{k l}, w\right)\right]} \\
& =\left[d_{i}\left(: \varepsilon_{i}(z) \varepsilon_{i}^{*}(z):-: \varepsilon_{i+1}(z) \varepsilon_{i+1}^{*}(z):\right),: \varepsilon_{k}(w) \varepsilon_{l}^{*}(w):\right] \\
& =\left(\alpha_{i} \mid \alpha_{k l}\right) X\left(\alpha_{k l}, z\right) \delta(z-w) .
\end{aligned}
$$

Serre relations are consequences of (3.7) and (3.9) for non-special nodes. It then follows that the operators $X\left( \pm \alpha_{i}, z\right)$ generate a type $A$ subalgebra for $i=1, \ldots, n-$ $1\left(n-2\right.$ for $\left.A_{n-1}\right)$. The verification for the special node 0 is quite similar to the above provided that we view $\beta$ as $\varepsilon_{1}$ in type $C_{n}$ and as $\varepsilon_{n}$ in type $A$ when checking commutation relations.

In types $D_{n}$ and $B_{n}$, the basic commutation relations are already given above using similar analysis to (3.7). For instance in type $B_{n}$ we have that

$$
\begin{aligned}
& {\left[X\left(\alpha_{0}, z\right), X\left(-\alpha_{1}, w\right)\right]} \\
& =\left[: \varepsilon_{0}(z) \varepsilon_{1}^{*}(z):-: \varepsilon_{\overline{1}}(z) \varepsilon_{\overline{0}}^{*}(z):,: \varepsilon_{2}(w) \varepsilon_{1}^{*}(w):-: \varepsilon_{\overline{1}}(w) \varepsilon_{2}^{*}(w):\right]=0 .
\end{aligned}
$$

Serre relations can be easily checked by using the above commutation relations plus properties of roots. We include one example to show the method. In the case of type $C_{n}$ we have

$$
\begin{aligned}
& {\left[X\left(\alpha_{0}, z_{1}\right),\left[X\left(\alpha_{0}, z_{2}\right), X\left(\alpha_{1}, w\right)\right]\right]=\frac{1}{2}\left[X\left(\alpha_{0}, z_{1}\right),\left[: \beta^{*}\left(z_{2}\right) \varepsilon_{1}^{*}\left(z_{2}\right):,: \varepsilon_{1}(w) \varepsilon_{2}^{*}(w):\right]\right]} \\
& =\frac{1}{4}\left[: \beta^{*}\left(z_{2}\right) \varepsilon_{1}^{*}\left(z_{2}\right):,: \beta^{*}\left(z_{2}\right) \varepsilon_{2}^{*}\left(z_{2}\right):+: \varepsilon_{1}^{*}\left(z_{2}\right) \varepsilon_{2}^{*}\left(z_{2}\right):\right] \delta\left(z_{2}-w\right)=0, \\
& \quad\left[X\left(\alpha_{1}, z_{1}\right),\left[X\left(\alpha_{1}, z_{2}\right),\left[X\left(\alpha_{1}, z_{3}\right), X\left(\alpha_{0}, w\right)\right]\right]\right] \\
& \quad=\frac{1}{2}\left[X\left(\alpha_{1}, z_{1}\right),\left[X\left(\alpha_{1}, z_{2}\right),\left[: \varepsilon_{1}\left(z_{3}\right) \varepsilon_{2}^{*}\left(z_{3}\right):,: \beta^{*}(w) \varepsilon_{1}^{*}(w):\right]\right]\right] \\
& \quad=-\frac{1}{2}\left[X\left(\alpha_{1}, z_{1}\right),\left[: \varepsilon_{1}(z) \varepsilon_{2}^{*}\left(z_{2}\right):,\left[: \varepsilon_{2}^{*}(w) \beta^{*}(w):+: \varepsilon_{2}^{*}(w) \varepsilon_{1}^{*}(w):\right]\right] \delta\left(z_{3}-w\right)\right. \\
& \quad=\left[X\left(\alpha_{1}, z_{1}\right),: \varepsilon_{2}^{*}(w) \varepsilon_{2}^{*}(w):\right] \delta\left(z_{2}-w\right) \delta\left(z_{3}-w\right)=0 .
\end{aligned}
$$

The toroidal Lie algebra of type $A_{1}$ is also realized by the same formulae as given above. However some commutation relations are different from those for $A_{n-1}(n \geq 3)$ :

$$
\begin{gathered}
{\left[X\left(\alpha_{0}, z\right), X\left(\alpha_{1}, w\right)\right]=\left(: \varepsilon_{2}(w) \varepsilon_{2}^{*}(w):-: \beta^{*}(w) \varepsilon_{1}(w):\right) \delta(z-w)-\partial_{w} \delta(z-w),} \\
{\left[X\left(-\alpha_{0}, z\right), X\left(-\alpha_{1}, w\right)\right]=\left(: \beta(w) \varepsilon_{1}^{*}(w):-: \varepsilon_{2}(w) \varepsilon_{2}^{*}(w):\right) \delta(z-w)-\partial_{w} \delta(z-w),} \\
{\left[X\left(\alpha_{0}, z_{1}\right),\left[X\left(\alpha_{0}, z_{2}\right), X\left(\alpha_{1}, w\right)\right]\right]=-2: \beta^{*}(w) \varepsilon_{2}(w): \delta\left(z_{1}-w\right) \delta\left(z_{2}-w\right),} \\
{\left[X\left(\alpha_{1}, z_{1}\right),\left[X\left(\alpha_{1}, z_{2}\right), X\left(\alpha_{0}, w\right)\right]\right]=-2 X\left(\alpha_{1}, w\right) \delta\left(z_{1}-w\right) \delta\left(z_{2}-w\right),}
\end{gathered}
$$

where the last two relations imply the Serre relations immediately for a toroidal Lie algebra of type $A_{1}$. We remark that the construction for type $B_{2}$ gives another representation of a toroidal Lie algebra of type $C_{2}$ at level -2 . 


\section{REFERENCES}

[BBS] S. Berman, Y. Billig, J. Szmigielski, Vertex operator algebras and the representation theory of toroidal algebras. Recent developments in infinite-dimensional Lie algebras and conformal field theory (Charlottesville, VA, 2000), 1-26, Contemp. Math., 297, Amer. Math. Soc., Providence, RI, 2002. MR,1919810 (2003j:17037)

[B] Y. Billig, Principal vertex operator representations for toroidal Lie algebras. J. Math. Phys. 39 (1998), no. 7, 3844-3864. MR1630546 (99j:17041)

[FF] A. Feingold, I. B. Frenkel, Classical affine algebras. Adv. Math. 56 (1985), 117-172. MR.788937 (87d:17018)

[FJW] I. Frenkel, N. Jing, W. Wang, Vertex representations via finite groups and the McKay correspondence. Int. Math. Res. Notices 4 (2000), 195-222. MR1747618 (2001c:17042)

[G] Y. Gao, Fermionic and bosonic representations of the extended affine Lie algebra $\mathfrak{g l}_{N}\left(\mathbb{C}_{q}\right)$. Canad. Math. Bull. 45 (2002), no. 4, 623-633. MR1941230(2003h:17030)

[JMg] C. Jiang, D. Meng, Vertex representations for the $\nu+1$-toroidal Lie algebra of type $B_{l}$. J. Algebra 246 (2001), no. 2, 564-593. MR.1872115 (2003b:17036)

[JM] N. Jing, K. C. Misra, Fermionic realization of toroidal Lie algebras of types ABD, arXiv:0807.3056.

[K] V. G. Kac, Vertex algebras for beginners. Univ. Lecture Ser., 10, Amer. Math. Soc., Providence, RI, 1997. MR1417941 (99a:17027)

[L] M. Lau, Bosonic and fermionic representations of Lie algebra central extensions. Adv. Math. 194 (2005), no. 2, 225-245. MR2139913(2005m:17008)

[MRY] R. V. Moody, S. E. Rao, T. Yokonuma, Lie algebras and Weyl groups arising from vertex operator representations. Nova J. Algebra Geom. 1 (1992), no. 1, 15-57. MR.1163780 (93h:17040)

[T] S. Tan, Vertex operator representations for toroidal Lie algebra of type $B_{l}$. Comm. Algebra 27 (1999), no. 8, 3593-3618. MR.1699582 (2001a:17041)

School of Sciences, South China University of Technology, Guangzhou 510640, PeoPle's Republic of China - And - Department of Mathematics, North Carolina State University, Raleigh, North Carolina 27695-8205

E-mail address: jing@math.ncsu.edu

Department of Mathematics, North Carolina State University, Raleigh, North CaroLINA 27695-8205

E-mail address: misra@math.ncsu.edu

School of Mathematics and Information Science, Wenzhou University, Wenzhou 325035, People's Republic of China

E-mail address: xuchongbin1977@126.com 